\title{
1 Is the habit system altered in individuals with obesity? A systematic review
}

Luis F. Ciria ${ }^{1,2^{*}}$ Poppy Watson ${ }^{3}$, Miguel A. Vadillo ${ }^{1} \&$ David Luque ${ }^{1,2^{*}}$

${ }^{1}$ Departamento de Psicología Básica, Universidad Autónoma de Madrid, ES

${ }^{2}$ Departamento de Psicología Básica, Universidad de Málaga, ES

${ }^{3}$ School of Psychology, University of New South Wales, Sydney, Australia

8

*Corresponding authors:

\section{Luis F. Ciria}

Departamento de Psicología Básica

Address: C/ Ivan Pavlov, 6.

28049. Madrid

Phone: +34 667876160

Email: 1ciria@uma.es

\section{David Luque}

Departamento de Psicología Básica

Address: C/ Ivan Pavlov, 6.

28049. Madrid

Mail: david.luque@gmail.com

\section{ABSTRACT}

Habit-like eating behavior is repeatedly pointed to as a key cognitive mechanism contributing to the emergence and maintenance of obesity. Here, we conducted a systematic review of the literature to assess the existent behavioral evidence for the Habit Hypothesis for Overeating (HHO) which states that obesity is the consequence of an imbalance between the habit and goal-directed reward learning systems, leading to overconsumption of food. We found a total of 19 studies implementing a variety of experimental protocols (i.e., free operant paradigm, slips-of-action test, two-step task, Pavlovian-to-Instrumental paradigm, probabilistic learning task) and manipulations. Taken together, the studies on clinical (binge eating disorder) and non-clinical individuals with overweight or obesity, do not support the HHO conclusively. While the scientific literature on HHO is still in its infancy, the heterogeneity of the extant studies makes it difficult to evaluate the degree of convergence of these findings. Uncovering the role of reward learning systems in eating behaviors might have a transformative impact on public health. 


\section{INTRODUCTION}

47 Obesity is an increasing pandemic with major repercussions in general and special population health, and a burden for health systems all around the world (Swinburn et al., 2011; Hales et al., 2018). Many different factors are involved in the development and maintenance of obesity, both of a biological (e.g. energy requirements, body weight, gastrointestinal signals, etc.) and of a non-biological nature (e.g., emotions, social environment, cognitive, etc.). These factors may increase body weight through a decrease in energy expenditure or an increase in energy intake. Thus, correctly understanding overeating - the main factor responsible for an increase in energy intake - is a crucial and pressing research issue. The surge in research on this topic in recent decades has provided valuable insight into overeating. However, the neuropsychological determinants for this behavior are still poorly understood. Indeed, psychological interventions do not reliably produce stable long-term reductions in excessive eating (Castelnuovo et al., 2017), and prevention programs have not shown reliable results either (Stice et al., 2006).

Individuals with obesity usually express the desire to limit food consumption but nevertheless persist overeating despite being aware of the negative consequences, suggesting that overeating and addiction may share some cognitive features. The similarities between obesity and addiction have led to theoretical and empirical work translating models and protocols from addiction to obesity research (Stice and Yokum, 2016). One of the hypotheses from addiction research that has influenced the study of obesity most is that problematic and persistent behaviors (such as drug consumption or overeating) are the result of an imbalance between the habit and the goal-directed reward learning systems (Ostlund and Balleine, 2008). This hypothesis, which we will refer to as the Habit Hypothesis for Overeating (HHO), claims that overweight and obesity are the consequence of predominant habit-like eating behavior, 
systems, leading the person to lose control over their eating behavior. The persistent nature of overeating behaviors would be explained if overeating and hence obesity are, at least partly, produced by over-reliance on habit-like unhealthy eating behaviors. More importantly, by understanding the role of habits in eating behavior, we could use what we already know about the habit system to design more successful weight loss interventions and obesity prevention campaigns. In this article, we review and assess the body of evidence supporting the HHO.

Drug addiction research seems to provide indirect support for the $\mathrm{HHO}$, pointing to a possible alteration of neural and cognitive dynamics related to habits and goal-directed reward learning systems in obesity. It has been repeatedly stated that obesity and drug addictions exhibit neural, cognitive and behavioral commonalities, since both phenomena can be conceptualized as substance abuse disorders (Kenny, 2011; Volkow and Wise, 2005). These commonalities are especially prominent in binge eating disorder (BED), a clinical eatingrelated condition characterized by the presence of recurrent episodes of compulsive eating in the absence of regular compensatory behaviors such as vomiting or laxative abuse (see Dingemans et al., 2002, for a review). BED usually co-occurs with overweight or obesity (e.g., de Zwaan, 2001) and is often considered as a food addiction because symptoms meet substance dependence disorder criteria (e.g., Cassin and von Ranson, 2007). In fact, similar alterations in brain regions and neural dynamics have been frequently reported both in individuals with obesity (with and without BED) and individuals with drug addictions (Wang et al., 2001; Volkow and Wise, 2005; Wong et al., 2006; Sjoerds et al., 2013; Tomasi et al., 2015; Volkow et al., 2008). These common alterations suggest that although food- and drug-consumption may be initially goal-directed behaviors in which these stimuli (food or drug) are voluntarily sought and taken for their rewarding effects, both can evolve into maladaptive stimulus-response (SR) habits (Everitt and Robbins, 2005; Belin and Everitt, 2008). However, it is important to highlight that obesity, BED and drug-addiction are multifactorial phenomena, with markedly 
different etiology and psychophysiological underlying mechanisms. Consequently, any parallels between them should be qualified regarding the level of explanation at which they are addressed.

We focused the current review to those studies in which the behavior under scrutiny was de-novo learned in the laboratory (i.e., studies within the framework of associative and reward learning literature). Even within this narrower literature, the existing studies that have directly addressed the HHO so far have used very different methodologies to assess the habit/goal-directed nature of behavior. Studies also differ in terms of how the results of the habit test (whatever is used) are related with overeating and obesity. Finally, their results are not always consistent and different approaches seem to lead to different conclusions.

In the current review, we included all studies that claimed to measure the habit/goaldirected component of behavioral responses, rather than exclude studies that did not, in our opinion, measure habits/goal-directed behavior in an appropriate manner. We have left a more detailed discussion of these concerns for the final part of this review and believe by including a wider range of studies, that the reader can have a more complete and unbiased view of this literature base.

In general, all the studies included in this review define the goal-directed component of behavioral responses as the flexible adaptation of responses after some training: inflexibility (for instance, persistent responses to devalued outcomes) is taken as a proxy of habitualization of that behavior. This general strategy has been applied through different experimental protocols, which have been used to organize the review and guide the article search process. However, we must note here that the canonical definition of habits establishes that habits relied on cached S-R associations: The ongoing value of the outcome has no power for modulating an already existing habit (since it is outside the S-R memory). As a consequence, habits are defined as responses that are not changed (in frequency and/or intensity) even when (1) the 
outcome following that response is no longer desired or (2) that response is no longer effective in producing the outcome (Dickinson, 1985). Conversely, goal-directed responses are those which change given the aforementioned circumstances. As we will see, only experiments using an outcome devaluation test can be argued to meet the first criterion and assess the balance of these learning systems in line with the canonical approach (habits can be also studied by using a contingency degradation test, which relies on the second criterion, but this test is seldom used in human research).

\subsection{Experimental protocols to measure the habit/goal-directed component of a response.}

The outcome devaluation paradigm is considered the gold standard paradigm to dissociate goal-directed actions from habits in eating behaviors in humans. This paradigm usually consists of three phases: $(i)$ training subjects in two instrumental actions leading to two different food outcomes, (ii) devaluation of one of the two food outcomes, (iii) measurement of the response to the outcome that has been previously devalued. If participants show a reduced frequency of the action associated with the now devalued outcome it suggests that behavior is goal-directed, while the absence of this behavioral sensitivity would indicate habitual behavior (Dickinson et al., 1995). The devaluation phase hence plays a crucial role to properly dissociate goal-directed actions from habits. Satiation has been frequently used to devalue food outcomes, by allowing ad libitum access to food reward at the devaluation phase (Parkes et al., 2016). Researchers measure the magnitude of difference between responses for the stimulus that predict the devalued (sated) food outcome before and after satiation, compared with a stimulus that predicts a non-devalued food outcome. This selective satiation procedure allows researchers to obtain an index of the balance between goal-directed and habitual systems. investigated using reinforcement-learning tasks that do not contain a devaluation procedure. 
Instead, by implementing sequential decision-making tasks combined with computational

147 modelling algorithms, researchers have tried to dissociate the relative influence of goal-

148 directed and habit systems on choice behavior (Daw et al., 2011). The common procedure of this paradigm consists of two stages with binary response choices. In the first stage, participants are instructed to choose between a stimulus-pair that leads, with fixed probability, to one of two stimuli-pairs in a second stage. Stimulus selection in the second stage leads to a reward with probability varying gradually over time according to a random walk. Learning strategy is inferred by estimating the degree to which the reinforcement of participant's previous choices leads to a mere repetition of responses (i.e., model-free strategy), and the degree to which participants integrate the likelihood of transitioning to a specific stimuli-pair in the second stage together with the feedback from the previous choice reinforcement (i.e., model-based strategy). Thus, the model-free strategy depends on the likelihood with which participants choose a firststage stimulus depending on the previous trial outcome, while model-based strategy is reflected in the interaction between previous outcome and the transition between the stages of the task. The model-free strategy has been argued to reflect habit-like control, whereas the model-based strategy has been related to goal-directed behavior (Daw et al., 2005; Decker et al., 2016). In addition to these parameters, the relative balance of model-based and model-free strategies can be measured using the weighting parameter $(\omega)$, which seems to correlate with sensitivity to outcome devaluation (Friedel et al., 2014; Gillan et al., 2015) and performance on the slips-ofaction task (Sjoerds et al., 2016). On the other hand, independent measures of habit formation - obtained from an outcome devaluation test- do not correlate with model-free learning (Gillan et al., 2015). Thus, the parameter $\omega$ is probably indexing the functioning of the goaldirected system (see the Discussion section).

An indirect approach to measuring habits in eating behavior is the Pavlovian-toinstrumental transfer (PIT) task (see Cartoni et al., 2016 for a detailed review). In a classic 
outcome-specific PIT paradigm, Pavlovian associations between cues and food rewards and instrumental actions for those same food rewards are trained in separate experimental phases. For example, hungry participants might first learn to associate specific cues with the delivery of potato chips and chocolate before learning to make specific responses in order to earn potato chips and chocolate. The instrumental actions are then tested both in the presence and in the absence of the Pavlovian cues (Cartoni et al., 2016). Outcome-specific PIT refers to the behavioral phenomenon of increased instrumental responding for a reinforcer when in the presence of conditioned-stimulus (CS) that was previously paired with that same reinforcer (i.e. increased pressing for chocolate over chips in the presence of the chocolate CS). This outcome-specific transfer effect, which has been replicated with different food rewards in animals, as well as in humans (Cartoni et al., 2016), is observed even when the signaled food outcomes are no longer desired and is considered therefore 'habit-like' in nature (Holland, 2004; Watson et al., 2014). Furthermore, although PIT is argued to be a relatively inflexible response-priming behavior that is not sensitive to shifts in outcome value (Watson et al., 2018), others argue that PIT is, at least in humans, a goal-directed behavior that is sensitive to outcome devaluation (Mahlberg et al., 2019).

Taking a different approach, probabilistic learning paradigms have been often implemented to investigate potential deficits in reward learning systems. In these tasks, participants learn to associate abstract cues with different outcomes based on (probabilistic) trial-by-trial feedback, which allows researchers to investigate associative learning after positive and negative feedback in a stream of conflicting trials, as well as to measure the ability of participants to adapt their behavior when the contingencies of the task change abruptly (probabilistic reversal learning tasks). A failure to adapt behavior after reversal might suggest an over-reliance on inflexible, habitual control rather than goal-directed action control. In a food-related context, an impairment of individuals' ability to adjust ongoing eating behavior in 
the face of changing circumstances (e.g., satiation) with conflicting evidence (e.g., presence of food still on the table) would be a sign of the existence of an imbalance of goal-directed and habit systems in individuals with overweight and/or obesity. Notice that these paradigms cannot be considered as conclusive assessments of an imbalance between reward learning systems. Rather, these tasks may suggest a potential alteration of the goal-directed reward learning system that should be tested with more stringent paradigms (e.g., an outcome devaluation test).

To summarize: The objective of the present review is to critically assess the existent evidence for an imbalance between the habit and goal-directed systems in people with overweight and obesity. We also included individuals with BED as a relevant clinical model for testing the $\mathrm{HHO}$ because of its compulsive-like eating-behavior features and comorbidity with overweight and obesity; specifically, we assess whether markers for behavior habitualization are increased for non-clinical and clinical (BED) individuals with overweight or obesity as compared with control individuals with healthy weight.

\section{METHODS}

We conducted a systematic literature review following the Preferred Reporting for Items for Systematic Reviews and Meta-Analyses (PRISMA) guidelines (Moher et al., 2009).

\subsection{Literature search}

On April 15, 2021, the electronic databases PubMed and PsycInfo were searched for relevant studies by a string that combined the following terms: "((obesity) OR (obese) OR (overweight) OR (binge eating) OR (BED)) AND ((goal-directed) OR (habit) OR (habits) OR (devaluation) OR (Pavlovian-to-instrumental transfer) OR (model-free) OR (model-based) OR (reversal))”. The literature search was narrowed to the title of original published studies with human subjects 
and written in English language. Additional titles were identified by a manual search of reference sections of topic-relevant papers and citations to them by other papers.

223

\subsection{Eligibility criteria}

225

226

227

228

229

230

231

232

233

234

235

236

237

238

239

240

241

242

243

244

245

Studies were considered eligible for inclusion if they met the following criteria: $(i)$ laboratorybased experiments; ( $i$ i) new operant behaviors (habits) learned during the experiment; (iii) habit formation measured behaviorally (pure questionnaire studies were excluded); (iv) peerreviewed papers or preprints with a DOI; (v) non-medicated population; (vi) weight status considered as a factor in the analysis of the behavior indexing habit strength; (vii) adult age (children and older adults excluded). We did not establish any restrictions on publication date.

\subsection{Study selection}

After conducting electronic database searches, we pooled results and removed duplicate articles by using custom Matlab's scripts to identify similar titles of the articles that were later checked and manually removed. Next, articles with irrelevant titles and abstracts were excluded. Then, the excluded articles were examined by the last author (DL) to double-check their relevance for this review (none of the excluded was included again in the pool of relevant articles). Finally, the full-text articles of the remaining entries were retrieved and reviewed (see flowchart in Fig. 1 for details). The first (LC) and the last author (DL) assessed and judged the full texts of potentially relevant studies with respect to eligibility. In case of disagreement, the second author (PW) was consulted to reach consensus.

Relevant information from all the included studies was retrieved by the first author using a data extraction table form developed a priori (see Table 1). Extracted data include (i) main aim of the study; (ii) characteristics of the study population (e.g., number of participants, weight status); (ii) experimental paradigm; (iii) type of independent variable(s); (iv) type of 
246 outcome measure; (v) main findings. The last author conducted a reliability check of the final 247 articles to ensure the quality of the data extraction.

248

249 3. RESULTS

250 Our initial search resulted in 376 eligible records and we investigated an additional 56 studies

251 from our manual search (see Fig. 1). Then, we eliminated 98 duplicate records. From the 252 remaining 334 studies, we excluded 301 studies non-related with HHO, and 14 because they

253 did not fulfil eligibility criteria. In the end, 19 studies were included in this systematic review. 

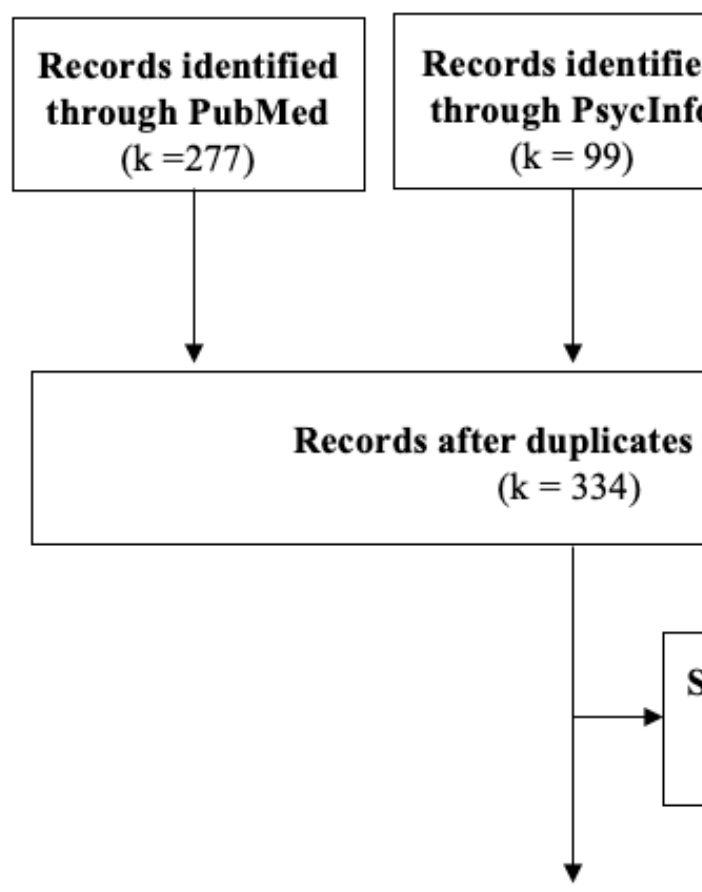

Studies excluded because titles or abstract were not relevant to HHO $(\mathrm{k}=301)$

Full-text articles assessed for eligibility $(\mathrm{k}=33)$
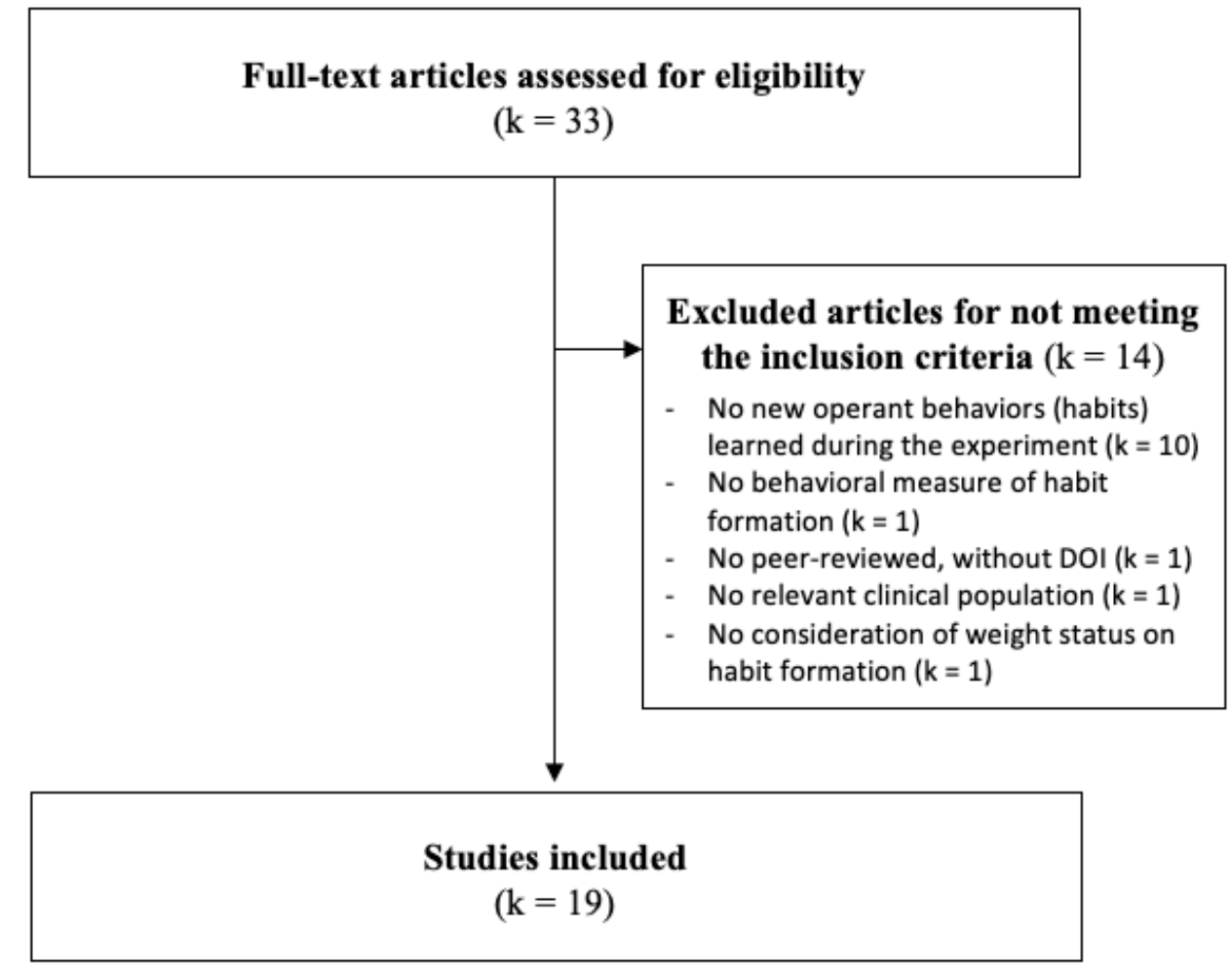

Figure 1: PRISMA flow chart for study inclusion.

3.1. Instrumental learning: devaluing outcomes to dissociate goal-directed actions from habits. 
Within the studies included in this systematic review, five relied on outcome devaluation paradigms to test the HHO (Horstmann et al., 2015; Dietrich et al., 2016; Janssen et al., 2017; Watson et al., 2017; Heriseanu et al., 2020). The best example of this approach is the seminal study conducted by Horstmann and collaborators (2015), in which a selective satiation procedure was implemented to investigate the differences in food-related goal-directed actions and habits associated with weight status in 26 young male adults. Participants were trained to associate two abstract stimuli (fractal images) with two food rewards (sweet, salty) by pressing two different buttons. After the initial training phase, participants had ad libitum access to one of the food rewards for $30 \mathrm{~min}$ while watching TV. Then, the response rate to both abstract stimuli was measured again. When the authors regressed the magnitude of the difference between pre and post responses to the devalued food outcome against weight status (measured by body mass index; BMI), they found that higher BMI led to an attenuated sensitivity to reward devaluation, compared with lower BMI. Interestingly, there were no differences as a function of BMI in self-reported motivational value of food outcomes pre and post satiation. In spite of this, Horstmann et al. concluded that high BMI might be associated with heightened habit-like responding for food outcomes, which could partially explain the difficulties of individuals with overweight and obesity to properly regulate eating behavior. A similar result was reported in a later study conducted by Jansen and collaborators using a four-choice satiety task as part of a larger fMRI study (Janssen et al., 2017). In line with Horstmann et al., they found that increased overweight was related to decreased goal-directed control of food choices following outcome devaluation.

While the aforementioned studies apparently support the HHO, some methodological issues should be taken into account. Firstly, in Horstmann et al., the choice test after satiation was not performed in extinction (i.e., participants continued receiving immediate feedback on 
order to prevent novel learning and examine instead the integration of the new outcome value with existing R-O associations (Adams and Dickinson, 1981). Differences therefore in performance in the test phase of Horstmann et al. between individuals with obesity and subjects with healthy weight, could be due to differences in new learning from (devalued) feedback rather than differences in habitual responding per se. Secondly, the reduced sample size of Horstmann et al. (i.e., 26 participants) compromises the statistical power of their regression analyses. That low statistical power not only reduces the chances of finding a significant result if it exists; it also reduces the positive predictive value (Button et al., 2013). Thirdly, the outcome devaluation phase did not seem to be effective in Janssen et al., since their participants still reported moderate feelings of hunger before the devaluation phase. Finally, in both studies participants were not explicitly told to eat the to-be-devalued food item until they felt full, instead they were simply instructed to eat as much of the target food item as they wanted. Consequently, participants could have adjusted food intake according to their experimental expectancies (Robinson et al., 2018) or social norms (Vartanian et al., 2013), compromising the reliability of the devaluation procedure, which fits with Horstmann et al. finding that selfreported motivational value did not seem to correlate with BMI.

In a recent study, Heriseanu and collaborators (2020) used a different method to devalue food outcomes within an instrumental learning paradigm. Whereas previous studies used selective satiation (Horstmann et al., 2015; Janssen et al., 2017), Heriseanu et al. devalued the food outcomes by requiring participants to watch a video of cockroaches making direct contact with the previously trained valuable food outcomes. In contrast with the aforementioned studies, they did not find significant differences in R-O devaluation in individuals with obesity compared with subjects with healthy weight. In addition, they included a third group of individuals with obesity and eating disorder features that exhibited a reduced behavioral 
disorders, and to the individuals with healthy weight. Notably, although these individuals with

311 obesity and eating disorder features were not properly diagnosed, the authors state that they could be broadly characterized as individuals with BED features, pointing to a potential imbalance between reward learning systems in individuals with obesity and BED.

Another method to devalue outcomes is through instructed devaluation as is used in the slips-of-action task (de Wit et al., 2012). In the outcome devaluation phase of this task, participants are instructed that some outcomes are no longer worth points (equivalent to food or monetary reward) and that responding to them will result in the loss of points. Participants are then instructed to quickly respond to a fast sequence of stimuli that predict valuable outcomes (e.g., food, money) and withhold responding when stimuli that predict devalued outcomes appear. A failure to refrain from responding to cues associated with devalued outcomes is interpreted as evidence for habitual control (Watson and de Wit, 2018). This approach has been followed by different authors to investigate the balance between goaldirected actions and habits in eating behaviors. For instance, Dietrich and collaborators (2016) recruited 105 participants, divided into three BMI groups (i.e., healthy-weight, overweight, obesity), to perform a slip-of-action task in a non-food context (i.e., money as reward). Contrary to what they expected, the results did not support the HHO and no relationship was found between weight status and sensitivity to devaluation. The authors pointed to a ceiling effect in task performance that could hide subtle differences in goal-directed actions control potentially associated with weight status (i.e., lack of task sensitivity). Similar results were found later by Watson and collaborators (2017) but, in this case, using a food-related slips-ofaction task (i.e., food as rewards). In that study, individuals with obesity did not make more slips of action in the presence of cues signaling devalued food outcomes, as compared with a healthy-weight control group. However, the reduced sample size $(n=38)$ and the betweengroup differences in the recruitment procedure (participants with obesity were in the process 
of preparing for bariatric surgery to reduce their BMI) strongly limit the generalization of these findings.

In sum, it appears that findings from instrumental learning paradigms using the canonical test for habits -i.e., outcome devaluation- show inconclusive evidence for an overreliance on habitual responding in individuals with overweight and/or obesity when tested in the context of both food and monetary rewards. Neither findings from studies using outcome devaluation procedures based on satiation, nor the rest of the studies using other devaluation procedures support conclusively a hypothetical increased habit propensity in obesity (with or without BED).

3.2. Reinforcement learning: computational modelling algorithms to disentangle goaldirected from habitual responses.

347 We identified three studies addressing the HHO by means of reinforcement-learning tasks that did not contain a devaluation procedure itself (Voon et al., 2015; Reiter et al., 2017; Janssen et al., 2020). Implementing sequential decision-making tasks, combined with computational modelling algorithms, these studies estimated the relative influence of goal-directed (modelbased) and habit (model-free) strategies in participants' decision making. The first one, conducted by Voon and collaborators (2015), focused on disorders involving compulsions (i.e., methamphetamine addiction, alcohol dependence, obsessive-compulsive disorder, and BED). By using a two-step sequential decision-making task in a non-food context (i.e., money as reward) the authors found a bias towards model-free learning in compulsive disorders groups (i.e., methamphetamine and obsessive-compulsive disorder), including the group of individuals with obesity and BED. In particular, this study compared the parameter $\omega$ between groups and concluded that individuals with BED showed lower relative engagement of a model-based strategy during the task. Crucially, they also included two control groups in the study: a group 
of participants with obesity but without BED and a group of individuals with healthy weight.

361 Contrary to the HHO, these latter groups did not differ in their behavioral strategy. A similar

362

363

364

365

366

367 study was later conducted by Janssen and collaborators (2020) addressing this issue in a nonclinical sample of 90 participants divided into three BMI groups (healthy-weight, over-weight, obese). Performance in the same two-step sequential decision task, in a non-food context, showed that the group with obesity had a lower $\omega$ (i.e., reduced relative engagement of a modelbased strategy during the task) than groups with overweight and healthy weight, which is in contrast with Voon et al.'s results when comparing individuals with and without obesity. Further, exploratory analyses showed a negative relationship between BMI and $\omega$. Thus, despite both studies implementing the same sequential decision-making task, the differences in performance for the group with obesity and the group with healthy weight stand, at first glance, in contrast. This could be, at least in part, due to differences between the samples of the studies since the Janssen et al.'s group with obesity had a higher BMI (on average) than the sample of Voon et al. In fact, Jannsen et al. suggest that the participants with obesity of their study might fulfil criteria for BED, although they did not systematically check it. Thus, it is reasonable to speculate that the group of individuals with obesity in the study of Jannsen et al. was comparable to the BED group in Voon et al. which also showed reduced relative engagement of a model-based strategy during this task. These results do not support the HHO in individuals with overweight or obesity but point to individuals with BED as a potential population that might exhibit such imbalance between habit and goal-directed reward learning systems.

Computational modelling of choice behavior was also used by Reiter and collaborators (2017) to address the HHO on individuals with BED. When comparing the behavior of a group of BED patients to a control group (i.e., individuals with healthy weight) in a reward-guided decision-making task, they observed poor behavioral adaptation in individuals with BED, 
relative to their counterparts (individuals with obesity but without BED). However, such impaired behavioral performance was not attributed to an over-reliance on model-free response pattern (i.e., habit-like responding), but to heightened switching behavior.

In sum, reinforcement-learning tasks combined with computational modelling seem to lend some support to the HHO in individuals with obesity and BED, but not with obesity in the absence of BED. We note, however, that reduced use of a model-based strategy does not necessarily suggest reliance on habits; indeed, Reiter and colleagues argued that BED patients simply made noisier, less consistent choices. In line with this, a recent study argues that the model-free parameter in the classic two-step paradigm reflects confusion about the task structure rather than persistent, habitual responding per se (da Silva and Hare, 2020).

3.3. Pavlovian-to-instrumental transfer: biasing instrumental responses toward signaled outcomes.

Three different studies have sought to investigate sensitivity of instrumental responding to cues that signal food rewards as a function of weight status in humans, using outcome-specific PIT paradigms (Watson et al., 2017; Lehner et al., 2017; Meemken and Horstmann, 2019). In general, these studies have not found evidence suggesting differences between healthy-weight and individuals with obesity in the strength of the outcome-specific transfer effect. For instance, Meemken and Horstmann (2019) compared instrumental responding for a specific 404 juice reward (e.g., mango juice) in the presence of a Pavlovian cue previously associated with 405 that juice, relative to trials with presentations of a cue paired with a different flavored juice 406 (e.g., apple juice). Fifty-one participants were included, 25 of whom had severe obesity (BMI $407>35)$. Outcome-specific PIT was observed in that participants pushed more for the currentlysignaled juice reward than the other juice reward, but the size of this PIT effect did not differ 409 between the groups of individuals with obesity and the healthy-weight. Likewise, using a 
410 design with both high- and low-calorie food outcomes, Watson and colleagues (2017) reported

411 that responses made by severely-individuals with obesity $(n=16)$ were more biased by high-

412 calorie cues than low-calorie cues, whereas no such difference was observed for the age-and-

413 education matched, healthy-weight individuals $(n=16)$. However, overall, the group with

414 obesity did not show stronger response priming (outcome-specific PIT) in the presence of either

415 high- or low-calorie food cues compared to those in the healthy-weight group. It should be noted though that participants in this study were not earning real food rewards, so it is not clear what strategies participants were employing when responding in the test phase.

Finally, Lehner and collaborators (2017) investigated responding on a food reward vs. neutral key in the presence of cues signaling either food rewards or neutral outcomes. Participants were 17 severely-individuals with obesity and 20 age-and-education matched, healthy-weight controls. Similarly, to the aforementioned studies, an overall PIT effect was observed (i.e. responding more on a food-reward key than a neutral key in the presence of cues signaling food rewards relative to neutral outcomes) and the group with obesity did not show significantly stronger PIT relative to the healthy-weight group. However, this study also included participants with overweight $(\mathrm{n}=17)$ and reported, surprisingly, that they showed a stronger PIT effect using this paradigm, relative to both healthy-weight and subjects with obesity. It should be noted that unlike classic outcome-specific PIT studies, where participants respond on keys associated with various food and drink rewards (Meemken and Horstmann, 2019), participants in the study of Lehner and colleagues had the choice to respond on a noreward key or a chocolate key (Lehner et al., 2017). Participants responded more on the noreward key than the chocolate key in the presence of the Pavlovian cue signaling the no-reward outcome. That is, participants had the opportunity to respond for chocolate but chose not to on some trials. This raises the question of whether participants in this study understood the task 434 instructions and understood that they could, in principle, respond on the chocolate key (rather 
than the no-reward key) on every trial, regardless of which cue was currently being presented. The strategies employed by participants in PIT tasks have been shown to be extremely sensitive to instruction, particularly instructions around the appearance of the Pavlovian cues in the test phase (Mahlberg et al., 2021). As such, the results of Lehman et al. should be interpreted with caution. In addition, none of the aforementioned PIT studies included outcome devaluation and therefore cannot be considered as a direct test of habit-like responding for devalued food outcomes.

Regardless of potential issues with task design, none of these studies —using varied designed with different food and drink outcomes — suggested evidence of increased outcomespecific PIT in participants with obesity relative to healthy-weight individuals. Therefore, although PIT paradigms have been considered, with some limitations (Corbit et al., 2007), as a useful complement to research into habits, to date, the existent evidence from PIT in eating behavior does not suggest that individuals with obesity are more sensitive to the biasing effect of cues signaling food rewards on ongoing goal-directed choices for food.

\subsection{Probabilistic learning: establishing associations in the face of uncertainty}

Probabilistic learning paradigms have been often used to test the HHO. We identified nine relevant studies using this approach (Davis et al., 2010; Horstmann et al., 2011; Danner et al., 2012; Mathar et al., 2017; Coppin et al., 2014; Zhang et al., 2014; Meemken et al., 2018; Kube et al., 2018; Sadler et al., 2020). The first three studies investigating a potential deficit of goaldirected actions control as a function of weight status implemented modified versions of the Iowa Gambling Task (IGT; Bechara et al., 1994). For instance, Davis and collaborators (2010) employed a case-[double] control design with three groups of women: with obesity $(n=73)$, with obesity and $\operatorname{BED}(n=65)$, and with healthy weight $(n=71)$. They found that the groups with obesity (with or without BED) exhibited poorer performance on the IGT, relative to their 
counterparts with healthy weight, but did not differ significantly from each other. Notably, the authors reported significantly lower educational levels, which was related to task performance, in the groups with obesity (with and without BED) compared to controls with healthy weight. Therefore, the impaired decision-making pattern observed in individuals with obesity may not be attributed to their weight status, but to their educational level. Nonetheless, later studies using the IGT have reported performance differences as a function of weight status in individuals with obesity (Horstmann et al., 2011) and BED (Danner et al., 2012). Particularly relevant is the study conducted by Horstmann and collaborators (2011) showing that BMI correlated with disadvantageous choices in the IGT, but only in women. Additionally, the authors reported several obesity-related structural brain differences between men and women in the left dorsal striatum and the right dorsolateral prefrontal cortex. Based on the functional relevance of these altered brain structures in habits and goal-directed action control, the authors hypothesized that eating behavior in individuals with obesity might be dominated by habit-like behavior as opposed to goal-directed actions. At this point, it is important to highlight that the

474 IGT cannot be considered as an appropriate paradigm to assess a possible imbalance between habit and goal-directed reward learning systems. While these results are relevant and suggest a relationship between obesity and decision-making, they can only suggest a potential alteration of the goal-directed reward learning system that must be confirmed with more conclusive paradigms (e.g., outcome devaluation test).

After the publication of the seminal work of Horstmann and collaborators in 2011, several studies have addressed the HHO using similar probabilistic learning tasks (Mathar et al., 2017; Coppin et al., 2014; Zhang et al., 2014; Meemken et al., 2018; Kube et al., 2018; Sadler et al., 2020). All these studies followed a common approach, which consisted of recruiting participants with a wide range of BMI values, usually dividing them into two 484 (healthy-weight, obese) or three groups (healthy-weight, overweight, obese), and instructing 
them to perform a probabilistic learning task. Task performance indices were then regressed against BMI or compared across groups. The results of these studies, with the exception of Meemken et al. (2018), indicate that, compared to their healthy-weight counterparts, individuals with obesity show systematic deficits in negative, but not in positive outcome learning, regardless of gender (Mathar et al., 2017; Coppin et al., 2014; Kube et al., 2018; Sadler et al., 2020). In addition, multiple regression analyses show a negative association between BMI and performance in probabilistic learning tasks (Mathar et al., 2017; Sadler et al., 2020). Importantly, this marked difference in probabilistic learning between individuals with healthy weight and individuals with obesity has been found not only when food is used as reinforcement (Mathar et al., 2017; Coppin et al., 2014; Zhang et al., 2014; Sadler et al., 2020), but also with money as reward (Mathar et al., 2017; Coppin et al., 2014; Kube et al., 2018). This finding is crucial because it suggests the absence of a context specificity of this learning deficit.

Together, evidence from probabilistic learning tasks suggests that obesity (with or without BED) might be linked to impaired reward-based associative learning and that this impairment might not be specific to the food domain. Note that these results cannot be directly attributed to strong habit formation or to weak goal-directed control. In addition, these findings rather point to a deficit in decision-making in individuals with obesity, which could be rooted in a difficulty to learn from negative outcomes. Whether this decision-making deficit may be considered a consequence of the gradual shift from goal-directed to habit-like eating behavior is something that the existing evidence from probabilistic learning tasks cannot directly address.

\footnotetext{
Table 1: Characteristic of the studies investigating the balance between the habit and the goal-directed reward learning systems.
} 


\begin{tabular}{|c|c|c|c|c|c|c|c|}
\hline Study & Method & $\begin{array}{l}\text { Samp } \\
\text { le }\end{array}$ & $\mathbf{N}$ & Age (SD)* & BMI (SD) & Outcome & Main Findings \\
\hline $\begin{array}{l}\text { Horstmann } \\
\text { et al., } 2015\end{array}$ & $\begin{array}{l}\text { Free } \\
\text { operant } \\
\text { paradigm }\end{array}$ & WR & 30 & $\begin{array}{r}25.9 \pm \\
{[19-30]}\end{array}$ & $\begin{array}{r}29.1 \pm \\
{[19-45]}\end{array}$ & Food & $\begin{array}{l}\text { BMI correlates negatively } \\
\text { with behavioral adjustment } \\
\text { after reward devaluation in } \\
\text { men. }\end{array}$ \\
\hline $\begin{array}{l}\text { Janssen et } \\
\text { al., } 2017\end{array}$ & $\begin{array}{l}\text { Free } \\
\text { operant } \\
\text { paradigm }\end{array}$ & WR & 76 & $31.5 \pm 10.7$ & $26.4 \pm 3.8$ & Food & $\begin{array}{l}\text { Obesity status }{ }^{\mathrm{a}} \text { correlates } \\
\text { negatively with behavioral } \\
\text { adjustment after reward } \\
\text { devaluation. }\end{array}$ \\
\hline $\begin{array}{l}\text { Heriseanu } \\
\text { et al., } 2020\end{array}$ & $\begin{array}{l}\text { Free } \\
\text { operant } \\
\text { paradigm }\end{array}$ & $\begin{array}{l}\mathrm{HW} \\
\mathrm{Ob} \\
\mathrm{BED}\end{array}$ & $\begin{array}{l}43 \\
25 \\
19\end{array}$ & $\begin{array}{r}27.8 \pm 7.5 \\
30.2 \pm 10.8 \\
28.2 \pm 8.2\end{array}$ & $\begin{array}{l}22.3 \pm 2.1 \\
33.9 \pm 3.1 \\
38.4 \pm 5.7\end{array}$ & Food & $\begin{array}{l}\mathrm{Ob}=\mathrm{HW} \text { and } \mathrm{BED}<\mathrm{Ob}- \\
\mathrm{HW} \text { in devaluation } \\
\text { sensitivity }\end{array}$ \\
\hline $\begin{array}{l}\text { Dietrich et } \\
\text { al., } 2016\end{array}$ & $\begin{array}{l}\text { Slips-of- } \\
\text { action } \\
\text { test }\end{array}$ & $\begin{array}{l}\mathrm{HW} \\
\mathrm{Ov} \\
\mathrm{Ob}\end{array}$ & $\begin{array}{l}36 \\
35 \\
34\end{array}$ & $\begin{array}{r}27.0[24.3- \\
29.8] \\
25.0[24.0- \\
28.0] \\
27.0[25.0- \\
29.3]\end{array}$ & $\begin{array}{c}21.7 \\
{[20.3-} \\
23.3] \\
26.8 \\
{[21.2-} \\
27.8] \\
33.1 \\
{[21.2-} \\
36.4]\end{array}$ & Money & $\begin{array}{l}\text { BMI does not correlate with } \\
\text { the devaluation sensitivity } \\
\text { index. }\end{array}$ \\
\hline $\begin{array}{l}\text { Watson et } \\
\text { al., } 2017\end{array}$ & $\begin{array}{l}\text { Slips-of- } \\
\text { action } \\
\text { test }\end{array}$ & $\begin{array}{l}\mathrm{HW} \\
\mathrm{Ob}\end{array}$ & $\begin{array}{l}19 \\
19\end{array}$ & $\begin{array}{l}45.0 \pm 14.0 \\
43.9 \pm 10.6\end{array}$ & $\begin{array}{l}23.0 \pm 1.6 \\
44.0 \pm 7.1\end{array}$ & Food & $\begin{array}{l}\mathrm{Ob}=\mathrm{HW} \text { slips of actions in } \\
\text { the presences of cues } \\
\text { signaling devalued food } \\
\text { outcomes. }\end{array}$ \\
\hline $\begin{array}{l}\text { Voon et al., } \\
2015\end{array}$ & $\begin{array}{l}\text { Two-step } \\
\text { task }\end{array}$ & $\begin{array}{l}\mathrm{HW} \\
\mathrm{Ob} \\
\mathrm{BED}\end{array}$ & $\begin{array}{l}93 \\
31 \\
31\end{array}$ & $\begin{array}{r}43.1 \pm 10.2 \\
44.2 \pm 9.4 \\
42.7 \pm 9.0\end{array}$ & $\begin{array}{l}23.5 \pm 2.9 \\
31.5 \pm 3.6 \\
34.9 \pm 5.5\end{array}$ & Money & $\begin{array}{l}\mathrm{BED}>\mathrm{Ob}-\mathrm{HW} \text { in biasing } \\
\text { toward model-free (habit) } \\
\text { acquisition. }\end{array}$ \\
\hline $\begin{array}{l}\text { Janssen et } \\
\text { al., } 2020\end{array}$ & $\begin{array}{l}\text { Two-step } \\
\text { task }\end{array}$ & $\begin{array}{l}\mathrm{HW} \\
\mathrm{Ov} \\
\mathrm{Ob}\end{array}$ & $\begin{array}{l}31 \\
29 \\
30\end{array}$ & $\begin{array}{l}26.9 \pm 3.3 \\
26.0 \pm 3.7 \\
27.8 \pm 3.8\end{array}$ & $\begin{array}{l}21.6 \pm 1.8 \\
26.9 \pm 1.3 \\
35.4 \pm 4.5\end{array}$ & Money & $\begin{array}{l}\mathrm{Ob}<\mathrm{Ov}-\mathrm{HW} \text { relying on } \\
\text { model-based control. }\end{array}$ \\
\hline $\begin{array}{l}\text { Reiter et } \\
\text { al., } 2017\end{array}$ & $\begin{array}{l}\text { Two-step } \\
\text { task }\end{array}$ & $\begin{array}{l}\mathrm{Ob} \\
\mathrm{BED}\end{array}$ & $\begin{array}{l}22 \\
22\end{array}$ & $\begin{array}{l}27.8 \pm 4.5 \\
29.0 \pm 9.4\end{array}$ & $\begin{array}{l}26.0 \pm 4.3 \\
28.3 \pm 6.6\end{array}$ & Money & $\begin{array}{l}\mathrm{BED}>\mathrm{Ob} \text { relying on } \\
\text { model-based control }\end{array}$ \\
\hline $\begin{array}{l}\text { Watson et } \\
\text { al., } 2017\end{array}$ & PIT & $\begin{array}{l}\text { HW } \\
\text { OB }\end{array}$ & $\begin{array}{l}19 \\
19\end{array}$ & $\begin{array}{l}45.0 \pm 14.0 \\
43.9 \pm 10.6\end{array}$ & $\begin{array}{l}23.0 \pm 1.6 \\
44.0 \pm 7.1\end{array}$ & Food & $\begin{array}{l}\mathrm{Ob}=\mathrm{HW} \text { in outcome- } \\
\text { specific PIT effect }\end{array}$ \\
\hline $\begin{array}{l}\text { Lehner et } \\
\text { al., 2017) }\end{array}$ & PIT & $\begin{array}{l}\mathrm{HW} \\
\mathrm{Ov} \\
\mathrm{Ob}\end{array}$ & $\begin{array}{l}20 \\
17 \\
17\end{array}$ & $\begin{array}{r}29 \pm 9 \\
30 \pm 8 \\
33 \pm 12\end{array}$ & $\begin{array}{l}21.9 \pm 1.6 \\
27.3 \pm 1.5 \\
36.9 \pm 5.1\end{array}$ & Food & $\begin{array}{l}\mathrm{Ov}>\mathrm{HW}-\mathrm{Ob} \text { in outcome- } \\
\text { specific PIT effect. }\end{array}$ \\
\hline
\end{tabular}




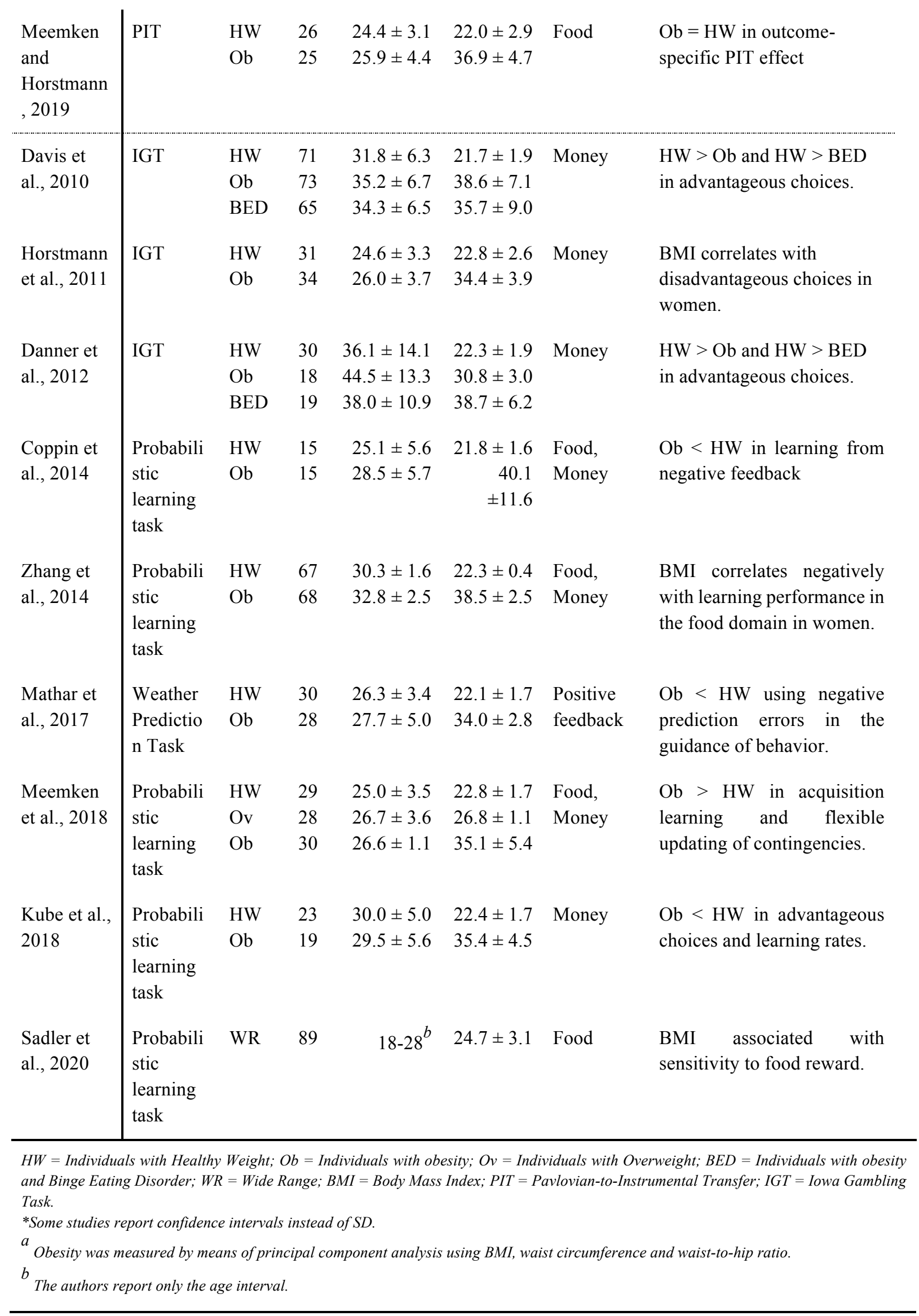




\section{DISCUSSION}

512 The purpose of the present study was to systematically review the existent empirical evidence

513 for the Habit Hypothesis for Overeating (HHO) that claims that overweight and obesity are the

514 consequence, at least partially, of an imbalance between the habit and goal-directed reward

515 learning systems, which leads to overconsumption of food. We found a total of 19 studies

516 addressing the HHO behaviorally. Taken together, these studies show non-consistent evidence

517 for an over-reliance on habitual responding in individuals with overweight and obesity in both

518 food and monetary reward contexts. In individuals with comorbid obesity and BED, the

519 existing studies suggest a potential imbalance between the reward learning systems, however,

520 the evidence is scarce (i.e., only two studies properly addressed the HHO in this population by using an outcome devaluation test). It is important to note that all the studies reviewed here followed cross-sectional or correlational approaches when testing the HHO, providing sparse and inconclusive evidence that, in any case, does not suggest causality.

In general, studies using outcome devaluation procedures based on satiation as well as studies using different outcome devaluation procedures (e.g., via instruction), provide inconsistent evidence for the $\mathrm{HHO}$, failing to show increased habitual eating behavior propensity in obesity. Partial support for the $\mathrm{HHO}$, albeit indirect, is provided by studies using probabilistic learning paradigms. Neither studies using sequential decision-making tasks nor studies based on PIT paradigms reveal the existence of an over-reliance on habitual responding in people with overweight or obesity (with or without BED). In short, the existing empirical evidence, to date, does not support the HHO conclusively. hence difficult to evaluate the degree of convergence of findings from studies that differ in how 
536 devaluation paradigm, considered as the gold standard for differentiating habits from goal537 directed actions (Parkes et al., 2016). When applied to eating behavior, and in particular for 538 testing the HHO, authors have implemented satiation (devaluation) procedures by allowing $a d$ 539 libitum access to a particular food reward (Horstmann et al., 2015; Janssen et al., 2017). This 540 eating-related outcome (food) devaluation procedure is mainly based on a physiological 541 attenuation of the ongoing food desire (motivational value) as a result of a feeling of fullness 542 that persists after eating, which is expected to suppress further consumption (Benelam, 2009).

543 Alternatively, others studies on HHO devalued food outcomes by changing their valence or 544 their magnitude (Dietrich et al., 2016; Watson et al., 2017; Heriseanu et al., 2020). For instance, 545 in the study of Heriseanu and collaborators, the authors diminished the motivational value of 546 food outcomes by their association with a negative event (i.e., food in direct contact with 547 cockroaches). This concomitant negative event shifts the valence of the food outcome and 548 attenuates its motivational value. However, others studies devalued the motivational value of 549 food outcomes by instructing participants that some outcomes were no longer worth points, 550 equivalent to food or monetary reward (Dietrich et al., 2016; Watson et al., 2017). That is, the 551 magnitude of the reward outcome is diminished or even suppressed, which attenuates its 552 motivational value. While all these outcome devaluation procedures have been extensively used to investigate the balance between habits and goal-directed actions (Gillan et al., 2016), they are considerably different in their nature when applied to eating behaviors. Because different outcome devaluation protocols have been used, it is not surprising that HHO's

556 literature reveals mixed evidence for a relationship between reward learning systems and obesity. Further, this is indicative that, any potential habit-like eating behavior in individuals with overweight or obesity would not be a generalized deficit, but specific to food- and satiation-related devaluation paradigms. In fact, the literature on compulsive disorders shows evidence for a domain general deficit, with overdominance of the habitual system over the 
goal-directed system (Gillan et al., 2016). In line with this, the existing evidence on individuals with obesity and BED seems to suggest a potential imbalance between both reward learning systems (Voon et al., 2015; Heriseanu et al., 2020), however, some paradigms used to address the $\mathrm{HHO}$ are not the most suitable and cannot directly prove this hypothesis. It is reasonable to speculate that individuals with obesity and compulsive traits, for example BED patients, would be more likely to exhibit habit-like eating behavior compared to individuals with overweight or obesity but without compulsive traits, although this is something to be assessed in the future, using more conclusive tests of habits.

Even assuming that all outcome devaluation protocols are effective, the reviewed literature still shows issues in the interpretation of their results. With the current protocols, habit and goal-directed systems are often defined reciprocally. That is, an individual who continues responding despite changes in the motivational value of a particular outcome might be interpreted as increased habit-like behavior, or to decreased goal-directed control, or even because of the regulation of both systems simultaneously (de Wit et al., 2018). In fact, it has been recently argued that habit and goal-directed systems might be organized hierarchically rather than in parallel (Botvinick et al., 2009; Dezfouli and Balleine, 2012), so that the formation of action sequences underlies habit learning and a goal-directed process selects between goal-directed actions and habitual sequences of actions to reach the goal (Dezfouli and Balleine, 2012). Thus, in the absence of a proper computational modelling approach to estimate the ongoing learning processes, the attribution of particular eating behaviors to a failure of a specific learning system (habitual or goal-directed) remains highly speculative. Nevertheless, computational modelling on sequential-decision tasks has also shown inconsistent results. In particular, recent accounts suggest that this approach may obscure enhanced habit-like patterns (Friedel et al., 2014; Gillan et al., 2015). Indeed, it has been reported that the strength of model-free learning is unrelated to habit formation and outcome 
insensitivity (Friedel et al., 2014; Gillan et al., 2015). In contrast, it seems that the model-free component of these models can be capturing the formation of incorrect models, rather than the operation of the habit system (da Silva and Hare, 2020). Thus, the standard two-step sequentialdecision task may not be sensitive enough to capture key processes involved in outcomeinsensitive habits, perhaps because participants have to choose between multiple outcomes with varying reward rates (Molinero et al., 2021; Wood and Rünger, 2016).

Human participants tend to use different (explicit) strategies that can potentially prevent the behavioral expression of habit formation (Watson and de Wit, 2018) or be confounded with true habits (da Silva and Hare, 2020). Indeed, behavioral paradigms addressing the HHO should be cautious when measuring habits and/or goal-directed behavior. A valid way to assure that a specific protocol is measuring habits and not goal-directed strategies is to assess how the responses in a test for habits (for instance, in an outcome devaluation test) develop with increasing training. Habits need frequent S-R pairings to be formed, while goal-directed strategies need just a few trials to be formed and are thought to operate at the beginning of learning (Dickinson et al., 1995; Adams, 1982; Dickinson and Weiskrantz, 1985). Thus, habits should be more evident in a group of participants with extensive training, while they should be easily overcome by the goal-directed system in a little trained control group. In other words, a valid protocol for measuring habits should show more habits with more training (Luque et al., 2020; Luque and Molinero, 2020). Worryingly, as reviewed here, this particular result has been evasive when the standard protocols for habits have been used (de Wit et al., 2018; Economides et al., 2015). In the framework of the HHO, none of the reviewed articles manipulated the amount of training and, indeed, the training phase was generally short ( 1 session of learning, when habit formation usually requires multiple sessions across multiple days of training) (Hardwick et al., 2019; Luque et al., 2020). Thus, it is not clear that the reviewed results were really measuring habit formation or goal-directed responses. 

bound behavior that is insensitive to devaluation (Watson and de Wit, 2018), however, when

613 directly addressing the HHO, this approach has not shown consistent effects (Lehner et al., 614 2017; Meemken and Horstmann, 2019; Watson et al., 2017). Nonetheless, three studies are not 615 sufficient evidence to rule out the HHO. In addition, recent accounts suggest that PIT could be sensitive to the devaluation of the outcome (Seabrooke et al., 2017) jeopardizing its reliability as an experimental model of habits.

Neuroimaging research on human eating behavior, albeit scarce, seems to provide partial support for the HHO. Several studies suggest that obesity might be associated with alterations in brain regions involved in habitual responding (Rothemund et al., 2007; Stoeckel et al., 2009; Horstmann et al., 2011; Nummenmaa et al., 2012; Babbs et al., 2013; Balodis et al., 2013; Mathar et al., 2017), as well as alterations in the dopamine and opiate systems, both related with goal-directed action control (Wang et al., 2001; Cosgrove et al., 2015; Guo et al., 2014; Kessler et al., 2014; van der Zwaal et al., 2016; Karlsson et al., 2015; Tuulari et al., 2017). In line with the HHO, these findings suggest that obesity (and likely the chronic consumption of obesogenic food) might be associated with alterations in neural dynamics and brain regions that control habits and goal-directed actions control. However, it is important to note that some of these experiments suffer from the same issues that we have identified above. points of the meal: before (i.e., conditioned satiety [e.g., Booth, 1977]), at the beginning (i.e., reward system with liking, preferences and aversions [e.g., Berridge, 1996]), after (i.e., classic satiety [e.g., Livingstone et al., 2000]) and, to a lesser degree, during the ingestion itself (i.e., specific sensory satiation [e.g., Rolls et al., 1981]). Most of the studies included in the present review assessed habits at the beginning of the meal or after it, therefore, there is still a gap in 
the literature on the potential influence of habits as a function of the timing of the meal that needs to be fully understood.

\subsection{Future directions}

While clear progress has been made in our understanding of the neuro-psychological factors contributing to persisting overeating, and particularly in the involvement of habit and goaldirected systems in eating behavior, a number of fundamental issues remain to be addressed.

At the top of the list is the establishment of a standard definition for what constitutes habit in eating behavior. As we aforementioned, the definition of habit is controversial and varies across research areas, which undermines current research on habits, and particularly on HHO (Gardner, 2015; Mazar and Wood, 2018; Gillan et al., 2016) Taking into account that the HHO suggests the existence of an imbalance between the habit and the goal-directed reward learning systems, it seems reasonable to conceptualize the habit within the general framework of associative and reward learning. Thus, a habit might be considered as an S-R association that operates independently of the ongoing value of its outcome, where the associated $\mathrm{R}$ is triggered whenever S is presented, even if the outcome is devalued and no longer desirable (de Wit and Dickinson, 2009). Additionally, it would be useful to add to the definition of habits the need of extensive training for their formation; this way, future research would consider to manipulate the amount of training in their designs. This standardization may not only facilitate comparisons and replication across studies but enhance the impact and validity of those studies interested in measuring the specific contribution of possible alterations of the habit system to obesity and overeating.

A second consideration, linked to the standard definition of habit, is the possibility that different experimental protocols might be more sensitive for measuring a potential imbalance between habit and goal-directed reward systems, and hence, more adequate for testing the 
HHO. As we have shown in this review, different paradigms have been used to operationalize

661

662

663

664

665

666

667

668

669

670

671

672

673

674

675

676

677

678

679

680

681

682

683

684

both the independent and dependent variables in HHO studies (i.e., free operant paradigm, slips-of-action test, two-step task, PIT, probabilistic learning task), but some of them do not provide a direct window on the extent to which the observed altered behavior patterns are due to strong habit formation or to weak goal-directed control (Watson and de Wit, 2018). Novel developments in the study of habits open interesting routes to test the HHO. The study of eating behavior in the form of a sequence, or chain, of linked behaviors (Balleine et al., 2015; Lingawi et al., 2016; Thrailkill et al., 2016); the degradation of instrumental contingencies between responses and outcomes, rendering the response unnecessary or counterproductive (Smith and Graybiel, 2014; Knowlton and Patterson, 2016); or the use of conditions in which the use of the goal-directed system is hindered (for instance, by reducing the time available to respond) (Hardwick et al., 2019; Luque et al., 2020); may constitute promising avenues to further understand the psychological determinants of overeating. The extent to which a reduced sensitivity to outcome devaluation in these paradigms can be attributed to an over-reliance on habit system or to poor goal-directed control largely remains to be investigated, maybe by manipulations of the amount of training. Regardless of the paradigm used, and in light of the studies reviewed here, it is reasonable to assume that this effect, if any, will be a small effect. It is hence imperative that future studies on HHO consider high-powered pre-registered tests of that hypothesis to increase sensitivity and fine-grained characterization of this effect and its potential moderators. In addition, in order to avoid relapsing into the classic paradox of null effects (i.e., the absence of evidence is not evidence of the absence), further studies might consider the use of Bayesian analytical approaches to assess the amount of evidence in favor of or against the proposed hypothesis (Dienes, 2014).

A third crucial issue is to further understand the brain and neural adaptations associated with overweight and obesity in humans; specifically, we need to explore the possible causal 
links between obesity, diet, and the reward learning system. Animal research may help to uncover these potential links, for example, through dietary intervention studies which are difficult to implement in human participants. For instance, Reichelt et al., (2014) investigated the effect of a highly palatable and caloric rich diet on rats' ability to learn about foodassociated cues. Those animals which received a high caloric diet persisted in responding to food-associated cues after outcome devaluation, while rats in the control group (normal diet) showed goal-directed adaptation of their responses. Similarly, Furlong et al., (2014) found that rats on a high caloric diet showed more habitual responses after outcome devaluation than control rats; this effect was mediated by differences in the dorsolateral striatum (i.e., a neural circuit that has been related to habitual control of behavior). This animal research illustrates how the adaptations arising from the continued exposure to unhealthy (high-fat, high-sugar) diets can play a causal role in of overweight and obesity through an unbalanced learning system. This body of research is crucial to further understand obesity and other eating-related disorders such as BED, all of which are also linked to maladaptive goal-directed actions control, and to plan more efficient interventions.

The fourth issue that we highlight in this review is the widespread use of BMI as an index of obesity. All studies included in this review, with the exception of Jansen et al (2017), used BMI to allocate individuals to the different weight status groups or to be regressed against habit/goal-directed indices. It is well known however that BMI is a poor index for obesity since it neither discriminates between lean body mass and fat mass nor reliably estimates the total fat or fat distribution (Rothman, 2008). Further, it does not necessarily indicate how healthy is the diet of an individual or how long a particular diet has been maintained, as BMI can be within the healthy range even though an individual has been on a high-fat diet for years. This poor sensibility of BMI to account for individual differences might explain, at least in part, the inconsistent results observed in the literature. The use of complementary measures of obesity 
such as the waist circumference or waist-to-hip ratio, as shown by Jansen et al,, or weight-

711 related measures as the individual and family history of obesity or the genetic background, may

712 allow a better characterization of individuals weight status and help detect possible subtle 713 effects.

714 It is also important to consider the wide range of factors that may influence food intake and eating behavior regulation. This myriad of factors at psychological (e.g., attitudes towards food, beliefs, mood, stress), social (e.g., incomes and educational level, food access) and cultural level (e.g., symbolism of the meal, role of the food in social meetings) may interfere with the persistence of overeating, in some cases being additive, but also in competition, revealing or hiding potential maladaptive adjustments between habit and goal-directed reward learning systems.

721

722

\subsection{Limitations}

723

The present systematic review is undermined by its narrow scope. By focusing only on the behavioral evidence for $\mathrm{HHO}$, only a restricted number of studies were included. The 19 studies that were included nonetheless used a wide range of paradigms and analytical approaches to address the $\mathrm{HHO}$, which drastically ruled out the possibilities of using meta-analysis to quantify the effect of a potential imbalance between reward-learning systems linked to obesity. Furthermore, many of the studies included were not designed to directly address the HHO (e.g., Horstmann et al., 2011; Watson et al., 2017), resulting in suboptimal study designs and analytical approaches for that purpose. Importantly, the lack of longitudinal data analyses on this topic (obesity/overweight), which is long-lasting and highly variable across time, drastically limits the reach of that research line.

\subsection{Final conclusion}


This systematic review sheds light onto the behavioral and cognitive mechanisms underlying overeating, showing no conclusive support an imbalance of reward learning systems in individuals with overweight and obesity (with and without BED). Despite the absence of conclusive evidence, it is important to highlight that the scientific literature on $\mathrm{HHO}$ is in its infancy. More research is needed to unveil the potential role of reward learning systems on the development and maintenance of obesity, as it could have a transformative impact on public health shifting the traditional framework in the cognitive-behavioral treatment of obesity towards habits as a key factor in weight loss programs and obesity prevention campaigns, which could be more effective than promoting self-control (Fürtjes et al., 2020).

\section{FUNDING}

L.F.C and D.L were supported by grant 2017-T1/SOC-5147 (Comunidad de Madrid) and grant

PGC2018-094694-B-I00 (AEI / FEDER, UE). M.A.V. was supported by grants 2016-T1/SOC1395 (Comunidad de Madrid), 2020-5A/SOC-19723 (Comunidad de Madrid) and PSI201785159-P (AEI, UE/FEDER).

\section{REFERNCES}

Adams, C.D., 1982. Variations in the sensitivity of instrumental responding to reinforcer devaluation. The Quarterly Journal of Experimental Psychology Section B 34, 77-98.

Adams, C.D., Dickinson, A., 1981. Instrumental Responding following Reinforcer Devaluation. The Quarterly Journal of Experimental Psychology Section B 33, 109-121. https://doi.org/10.1080/14640748108400816

Babbs, R.K., Sun, X., Felsted, J., Chouinard-Decorte, F., Veldhuizen, M.G., Small, D.M., 2013. Decreased caudate response to milkshake is associated with higher body mass index and greater impulsivity. Physiology \& behavior 121, 103-111.

Balleine, B.W., Dezfouli, A., Ito, M., Doya, K., 2015. Hierarchical control of goal-directed action in the cortical-basal ganglia network. Current Opinion in Behavioral Sciences 5, 1-7.

Balodis, I.M., Kober, H., Worhunsky, P.D., White, M.A., Stevens, M.C., Pearlson, G.D., Sinha, R., Grilo, C.M., Potenza, M.N., 2013. Monetary Reward Processing in Obese Individuals With and Without Binge Eating Disorder. Biological Psychiatry 73, 877-886. https://doi.org/10.1016/j.biopsych.2013.01.014

Bechara, A., Damasio, A.R., Damasio, H., Anderson, S.W., 1994. Insensitivity to future consequences following damage to human prefrontal cortex. Cognition 50, 7-15.

Belin, D., Everitt, B.J., 2008. Cocaine Seeking Habits Depend upon Dopamine-Dependent Serial Connectivity Linking the Ventral with the Dorsal Striatum. Neuron 57, 432-441. 
https://doi.org/10.1016/j.neuron.2007.12.019

Benelam, B., 2009. Satiation, satiety and their effects on eating behaviour. Nutrition bulletin 34, 126-173.

Berridge, K.C., 1996. Food reward: brain substrates of wanting and liking. Neuroscience \& Biobehavioral Reviews 20, 1-25.

Booth, D.A., 1977. Satiety and appetite are conditioned reactions. Psychosomatic Medicine 39, $76-81$. https://doi.org/10.1097/00006842-197703000-00002

Botvinick, M.M., Niv, Y., Barto, A.G., 2009. Hierarchically organized behavior and its neural foundations: a reinforcement learning perspective. Cognition 113, 262-280.

Button, K.S., Ioannidis, J.P., Mokrysz, C., Nosek, B.A., Flint, J., Robinson, E.S., Munafò, M.R., 2013. Power failure: why small sample size undermines the reliability of neuroscience. Nature reviews neuroscience 14, 365-376.

Cartoni, E., Balleine, B., Baldassarre, G., 2016. Appetitive Pavlovian-instrumental Transfer: A review. Neuroscience \& Biobehavioral Reviews 71, 829-848. https://doi.org/10.1016/j.neubiorev.2016.09.020

Cassin, S.E., von Ranson, K.M., 2007. Is binge eating experienced as an addiction? Appetite 49, 687-690.

Castelnuovo, G., Pietrabissa, G., Manzoni, G.M., Cattivelli, R., Rossi, A., Novelli, M., Varallo, G., Molinari, E., 2017. Cognitive behavioral therapy to aid weight loss in obese patients: current perspectives [WWW Document]. Psychology Research and Behavior Management. https://doi.org/10.2147/PRBM.S113278

Coppin, G., Nolan-Poupart, S., Jones-Gotman, M., Small, D.M., 2014. Working memory and reward association learning impairments in obesity. Neuropsychologia 65, 146-155. https://doi.org/10.1016/j.neuropsychologia.2014.10.004

Corbit, L.H., Janak, P.H., Balleine, B.W., 2007. General and outcome-specific forms of Pavlovian-instrumental transfer: the effect of shifts in motivational state and inactivation of the ventral tegmental area. European Journal of Neuroscience 26, 3141-3149.

Cosgrove, K.P., Veldhuizen, M.G., Sandiego, C.M., Morris, E.D., Small, D.M., 2015. Opposing relationships of BMI with BOLD and dopamine D2/3 receptor binding potential in the dorsal striatum. Synapse 69, 195-202.

da Silva, C.F., Hare, T.A., 2020. Humans primarily use model-based inference in the two-stage task. Nature Human Behaviour 4, 1053-1066.

Danner, U.N., Ouwehand, C., van Haastert, N.L., Hornsveld, H., de Ridder, D.T., 2012. Decision-making impairments in women with binge eating disorder in comparison with obese and normal weight women. European Eating Disorders Review 20, e56-e62.

Davis, C., Patte, K., Curtis, C., Reid, C., 2010. Immediate pleasures and future consequences. A neuropsychological study of binge eating and obesity. Appetite 54, 208-213.

Daw, N.D., Gershman, S.J., Seymour, B., Dayan, P., Dolan, R.J., 2011. Model-Based Influences on Humans' Choices and Striatal Prediction Errors. Neuron 69, 1204-1215. https://doi.org/10.1016/j.neuron.2011.02.027

Daw, N.D., Niv, Y., Dayan, P., 2005. Uncertainty-based competition between prefrontal and dorsolateral striatal systems for behavioral control. Nature Neuroscience 8, 1704-1711. https://doi.org/10.1038/nn1560

de Wit, S., Dickinson, A., 2009. Associative theories of goal-directed behaviour: a case for animal-human translational models. Psychological Research 73, 463-476. https://doi.org/10.1007/s00426-009-0230-6

de Wit, S., Kindt, M., Knot, S.L., Verhoeven, A.A.C., Robbins, T.W., Gasull-Camos, J., Evans, M., Mirza, H., Gillan, C.M., 2018. Shifting the balance between goals and habits: Five failures in experimental habit induction. Journal of Experimental Psychology: General 147, 1043-1065. https://doi.org/10.1037/xge0000402

de Wit, S., Watson, P., Harsay, H.A., Cohen, M.X., van de Vijver, I., Ridderinkhof, K.R., 2012. Corticostriatal connectivity underlies individual differences in the balance between habitual and goal-directed action control. Journal of Neuroscience 32, 12066-12075.

Decker, J.H., Otto, A.R., Daw, N.D., Hartley, C.A., 2016. From creatures of habit to goal-directed learners: Tracking the developmental emergence of model-based reinforcement learning. Psychological science 27, 848-858.

Dezfouli, A., Balleine, B.W., 2012. Habits, action sequences and reinforcement learning. European Journal of Neuroscience 35, 1036-1051. 
Dickinson, A., 1985. Actions and habits: the development of behavioural autonomy. Philosophical Transactions of the Royal Society of London. B, Biological Sciences 308, 67-78.

Dickinson, A., Balleine, B., Watt, A., Gonzalez, F., Boakes, R.A., 1995. Motivational control after extended instrumental training. Animal Learning \& Behavior 23, 197-206. https://doi.org/10.3758/BF03199935

Dickinson, A., Weiskrantz, L., 1985. Actions and habits: the development of behavioural autonomy. Philosophical Transactions of the Royal Society of London. B, Biological Sciences 308, 67-78. https://doi.org/10.1098/rstb.1985.0010

Dienes, Z., 2014. Using Bayes to get the most out of non-significant results. Frontiers in psychology 5, 781.

Dietrich, A., de Wit, S., Horstmann, A., 2016. General Habit Propensity Relates to the Sensation Seeking Subdomain of Impulsivity But Not Obesity. Front. Behav. Neurosci. 10. https://doi.org/10.3389/fnbeh.2016.00213

Dingemans, A.E., Bruna, M.J., Van Furth, E.F., 2002. Binge eating disorder: a review. International Journal of Obesity 26, 299-307.

Economides, M., Kurth-Nelson, Z., Lübbert, A., Guitart-Masip, M., Dolan, R.J., 2015. Model-based reasoning in humans becomes automatic with training. PLoS Comput Biol 11, e1004463.

Everitt, B.J., Robbins, T.W., 2005. Neural systems of reinforcement for drug addiction: from actions to habits to compulsion. Nature neuroscience 8, 1481-1489.

Friedel, E., n.d. Devaluation and sequential decisions: linking goal-directed and model-based behavior. Frontiers in Human Neuroscience 9.

Friedel, E., Koch, S.P., Wendt, J., Heinz, A., Deserno, L., Schlagenhauf, F., 2014. Devaluation and sequential decisions: linking goal-directed and model-based behavior. Frontiers in human neuroscience 8, 587.

Furlong, T.M., Jayaweera, H.K., Balleine, B.W., Corbit, L.H., 2014. Binge-like consumption of a palatable food accelerates habitual control of behavior and is dependent on activation of the dorsolateral striatum. Journal of Neuroscience 34, 5012-5022.

Fürtjes, S., King, J.A., Goeke, C., Seidel, M., Goschke, T., Horstmann, A., Ehrlich, S., 2020. Automatic and Controlled Processing: Implications for Eating Behavior. Nutrients 12, 1097. https://doi.org/10.3390/nu12041097

Gardner, B., 2015. A review and analysis of the use of 'habit' in understanding, predicting and influencing health-related behaviour. Health Psychology Review 9, 277-295. https://doi.org/10.1080/17437199.2013.876238

Gillan, C.M., Otto, A.R., Phelps, E.A., Daw, N.D., 2015. Model-based learning protects against forming habits. Cogn Affect Behav Neurosci 14.

Gillan, C.M., Robbins, T.W., Sahakian, B.J., van den Heuvel, O.A., van Wingen, G., 2016. The role of habit in compulsivity. European Neuropsychopharmacology, Compulsivity- a new trans-diagnostic research domain for the Roadmap for Mental Health Research in Europe (ROAMER) and Research Domain Criteria (RDoC) initiatives 26, 828-840. https://doi.org/10.1016/j.euroneuro.2015.12.033

Guo, J., Simmons, W.K., Herscovitch, P., Martin, A., Hall, K.D., 2014. Striatal dopamine D2-like receptor correlation patterns with human obesity and opportunistic eating behavior. Molecular psychiatry 19, $1078-1084$.

Hales, C.M., Fryar, C.D., Carroll, M.D., Freedman, D.S., Ogden, C.L., 2018. Trends in obesity and severe obesity prevalence in US youth and adults by sex and age, 2007-2008 to 2015-2016. Jama 319, 17231725.

Hardwick, R.M., Forrence, A.D., Krakauer, J.W., Haith, A.M., 2019. Time-dependent competition between goal-directed and habitual response preparation. Nature Human Behaviour 3, 1252-1262. https://doi.org/10.1038/s41562-019-0725-0

Heriseanu, A.I., Hay, P., Corbit, L., Touyz, S., 2020. Relating goal-directed behaviour to grazing in persons with obesity with and without eating disorder features. J Eat Disord 8, 48. https://doi.org/10.1186/s40337-020-00324-1

Holland, P.C., 2004. Relations Between Pavlovian-Instrumental Transfer and Reinforcer Devaluation. Journal of Experimental Psychology: Animal Behavior Processes 30, 104-117. https://doi.org/10.1037/00977403.30.2.104

Horstmann, A., Busse, F.P., Mathar, D., Müller, K., Lepsien, J., Schlögl, H., Kabisch, S., Kratzsch, J., 
Neumann, J., Stumvoll, M., Villringer, A., Pleger, B., 2011. Obesity-Related Differences between Women and Men in Brain Structure and Goal-Directed Behavior. Front. Hum. Neurosci. 5. https://doi.org/10.3389/fnhum.2011.00058

Horstmann, A., Dietrich, A., Mathar, D., Pössel, M., Villringer, A., Neumann, J., 2015. Slave to habit? Obesity is associated with decreased behavioural sensitivity to reward devaluation. Appetite 87, 175-183. https://doi.org/10.1016/j.appet.2014.12.212

Janssen, L.K., Duif, I., van Loon, I., Wegman, J., de Vries, J.H.M., Cools, R., Aarts, E., 2017. Loss of lateral prefrontal cortex control in food-directed attention and goal-directed food choice in obesity. NeuroImage 146, 148-156. https://doi.org/10.1016/j.neuroimage.2016.11.015

Janssen, L.K., Mahner, F.P., Schlagenhauf, F., Deserno, L., Horstmann, A., 2020. Reliance on model-based and model-free control in obesity. Scientific Reports 10, 1-14.

Karlsson, H.K., Tuominen, L., Tuulari, J.J., Hirvonen, J., Parkkola, R., Helin, S., Salminen, P., Nuutila, P., Nummenmaa, L., 2015. Obesity is associated with decreased $\mu$-opioid but unaltered dopamine D2 receptor availability in the brain. Journal of Neuroscience 35, 3959-3965.

Kenny, P.J., 2011. Common cellular and molecular mechanisms in obesity and drug addiction. Nat Rev Neurosci 12, 638-651. https://doi.org/10.1038/nrn3105

Kessler, R.M., Zald, D.H., Ansari, M.S., Li, R., Cowan, R.L., 2014. Changes in dopamine release and dopamine D2/3 receptor levels with the development of mild obesity. Synapse 68, 317-320.

Knowlton, B.J., Patterson, T.K., 2016. Habit formation and the striatum, in: Behavioral Neuroscience of Learning and Memory. Springer, pp. 275-295.

Kube, J., Mathar, D., Horstmann, A., Kotz, S.A., Villringer, A., Neumann, J., 2018. Altered monetary loss processing and reinforcement-based learning in individuals with obesity. Brain Imaging and Behavior 12, 1431-1449. https://doi.org/10.1007/s11682-017-9786-8

Lehner, R., 2017. Food-Predicting Stimuli Differentially Influence Eye Movements and Goal-Directed Behavior in Normal-Weight, Overweight, and Obese Individuals. Frontiers in Psychiatry 8, 15.

Lingawi, N.W., Dezfouli, A., Balleine, B.W., 2016. The psychological and physiological mechanisms of habit formation. The Wiley Handbook on the Cognitive Neuroscience of Learning 411-440.

Livingstone, M.B.E., Robson, P.J., Welch, R.W., Burns, A.A., Burrows, M.S., McCormack, C., 2000. Methodological issues in the assessment of satiety. Näringsforskning 44, 98-103.

Luque, D., Molinero, S., 2020. A critical assessment of the goal replacement hypothesis for habitual behaviour. Psicológica Journal 1.

Luque, D., Molinero, S., Watson, P., López, F.J., Le Pelley, M.E., 2020. Measuring habit formation through goal-directed response switching. Journal of Experimental Psychology: General 149, 1449-1459. https://doi.org/10.1037/xge0000722

Mahlberg, J., Seabrooke, T., Weidemann, G., Hogarth, L., Mitchell, C.J., Moustafa, A.A., 2021. Human appetitive Pavlovian-to-instrumental transfer: a goal-directed account. Psychological Research 85, 449463. https://doi.org/10.1007/s00426-019-01266-3

Mahlberg, J., Seabrooke, T., Weidemann, G., Hogarth, L., Mitchell, C.J., Moustafa, A.A., 2019. Human appetitive Pavlovian-to-instrumental transfer: a goal-directed account. Psychological research 1-15.

Mathar, D., Neumann, J., Villringer, A., Horstmann, A., 2017. Failing to learn from negative prediction errors: Obesity is associated with alterations in a fundamental neural learning mechanism. Cortex 95, 222237. https://doi.org/10.1016/j.cortex.2017.08.022

Mazar, A., Wood, W., 2018. Defining Habit in Psychology, in: Verplanken, B. (Ed.), The Psychology of Habit: Theory, Mechanisms, Change, and Contexts. Springer International Publishing, Cham, pp. 13-29. https://doi.org/10.1007/978-3-319-97529-0_2

Meemken, M.-T., Horstmann, A., 2019. Appetitive Pavlovian-to-Instrumental Transfer in Participants with Normal-Weight and Obesity. Nutrients 11, 1037. https://doi.org/10.3390/nu11051037

Meemken, M.T., Kube, J., Wickner, C., Horstmann, A., 2018. Keeping track of promised rewards: Obesity predicts enhanced flexibility when learning from observation. Appetite 131, 117-124.

Moher, D., Liberati, A., Tetzlaff, J., Altman, D.G., Group, T.P., 2009. Preferred Reporting Items for Systematic Reviews and Meta-Analyses: The PRISMA Statement. PLOS Medicine 6, e1000097. https://doi.org/10.1371/journal.pmed.1000097 
Molinero, S., Giménez-Fernández, T., López, F.J., Carretié, L., Luque, D., 2021. Stimulus-response learning and expected reward value enhance stimulus cognitive processing: An ERP study. Psychophysiology e13795.

Nummenmaa, L., Hirvonen, J., Hannukainen, J.C., Immonen, H., Lindroos, M.M., Salminen, P., Nuutila, P., 2012. Dorsal striatum and its limbic connectivity mediate abnormal anticipatory reward processing in obesity. PloS one 7, e31089.

Ostlund, S.B., Balleine, B.W., 2008. On habits and addiction: an associative analysis of compulsive drug seeking. Drug discovery today: Disease models 5, 235-245.

Parkes, S.L., Marchand, A.R., Ferreira, G., Coutureau, E., 2016. A time course analysis of satiety-induced instrumental outcome devaluation. Learning \& behavior 44, 347-355.

Reichelt, A.C., Morris, M.J., Westbrook, R.F., 2014. Cafeteria diet impairs expression of sensory-specific satiety and stimulus-outcome learning. Frontiers in psychology 5, 852.

Reiter, A.M., Heinze, H.-J., Schlagenhauf, F., Deserno, L., 2017. Impaired flexible reward-based decisionmaking in binge eating disorder: evidence from computational modeling and functional neuroimaging. Neuropsychopharmacology 42, 628-637.

Robinson, E., Bevelander, K.E., Field, M., Jones, A., 2018. Methodological and reporting quality in laboratory studies of human eating behavior. Appetite 125, 486-491. https://doi.org/10.1016/j.appet.2018.02.008

Rolls, B.J., Rolls, E.T., Rowe, E.A., Sweeney, K., 1981. Sensory specific satiety in man. Physiology \& Behavior 27, 137-142. https://doi.org/10.1016/0031-9384(81)90310-3

Rothemund, Y., Preuschhof, C., Bohner, G., Bauknecht, H.-C., Klingebiel, R., Flor, H., Klapp, B.F., 2007. Differential activation of the dorsal striatum by high-calorie visual food stimuli in obese individuals. Neuroimage 37, 410-421.

Rothman, K.J., 2008. BMI-related errors in the measurement of obesity. International journal of obesity 32, S56-S59.

Sadler, J.R., Shearrer, G.E., Papantoni, A., Gordon-Larsen, P., Burger, K.S., 2020. Behavioral and physiological characteristics associated with learning performance on an appetitive probabilistic selection task. Physiology \& Behavior 223, 112984. https://doi.org/10.1016/j.physbeh.2020.112984

Seabrooke, T., Le Pelley, M.E., Hogarth, L., Mitchell, C.J., 2017. Evidence of a goal-directed process in human Pavlovian-instrumental transfer. Journal of Experimental Psychology: Animal Learning and Cognition 43, 377-387. https://doi.org/10.1037/xan0000147

Sjoerds, Z., de Wit, S., van den Brink, W., Robbins, T.W., Beekman, A.T., Penninx, B.W., Veltman, D.J., 2013. Behavioral and neuroimaging evidence for overreliance on habit learning in alcohol-dependent patients. Translational psychiatry 3, e337-e337.

Sjoerds, Z., Dietrich, A., Deserno, L., de Wit, S., Villringer, A., Heinze, H.-J., Schlagenhauf, F., Horstmann, A., 2016. Slips of Action and Sequential Decisions: A Cross-Validation Study of Tasks Assessing Habitual and Goal-Directed Action Control. Front. Behav. Neurosci. 10. https://doi.org/10.3389/fnbeh.2016.00234

Smith, K.S., Graybiel, A.M., 2014. Investigating habits: strategies, technologies and models. Frontiers in behavioral neuroscience 8,39 .

Stice, E., Shaw, H., Marti, C.N., 2006. A meta-analytic review of obesity prevention programs for children and adolescents: the skinny on interventions that work. Psychological bulletin 132, 667.

Stice, E., Yokum, S., 2016. Neural vulnerability factors that increase risk for future weight gain. Psychological bulletin 142, 447.

Stoeckel, L.E., Kim, J., Weller, R.E., Cox, J.E., Cook, E.W., Horwitz, B., 2009. Effective connectivity of a reward network in obese women. Brain Research Bulletin 79, 388-395. https://doi.org/10.1016/j.brainresbull.2009.05.016

Swinburn, B.A., Sacks, G., Hall, K.D., McPherson, K., Finegood, D.T., Moodie, M.L., Gortmaker, S.L., 2011. The global obesity pandemic: shaped by global drivers and local environments. The Lancet 378, 804814.

Thrailkill, E.A., Trott, J.M., Zerr, C.L., Bouton, M.E., 2016. Contextual control of chained instrumental behaviors. Journal of Experimental Psychology: Animal Learning and Cognition 42, 401.

Tomasi, D., Wang, G.-J., Wang, R., Caparelli, E.C., Logan, J., Volkow, N.D., 2015. Overlapping patterns of 
brain activation to food and cocaine cues in cocaine abusers: association to striatal D2/D3 receptors. Human brain mapping 36, 120-136.

Tuulari, J.J., Tuominen, L., de Boer, F.E., Hirvonen, J., Helin, S., Nuutila, P., Nummenmaa, L., 2017. Feeding releases endogenous opioids in humans. Journal of Neuroscience 37, 8284-8291.

van der Zwaal, E.M., de Weijer, B.A., van de Giessen, E.M., Janssen, I., Berends, F.J., van de Laar, A., Ackermans, M.T., Fliers, E., la Fleur, S.E., Booij, J., 2016. Striatal dopamine D2/3 receptor availability increases after long-term bariatric surgery-induced weight loss. European Neuropsychopharmacology 26, 1190-1200.

Vartanian, L.R., Sokol, N., Herman, C.P., Polivy, J., 2013. Social models provide a norm of appropriate food intake for young women. PLoS One 8, e79268.

Volkow, N.D., Wang, G.-J., Telang, F., Fowler, J.S., Thanos, P.K., Logan, J., Alexoff, D., Ding, Y.-S., Wong, C., Ma, Y., 2008. Low dopamine striatal D2 receptors are associated with prefrontal metabolism in obese subjects: possible contributing factors. Neuroimage 42, 1537-1543.

Volkow, N.D., Wise, R.A., 2005. How can drug addiction help us understand obesity? Nat Neurosci 8, 555-560. https://doi.org/10.1038/nn1452

Voon, V., Derbyshire, K., Rück, C., Irvine, M.A., Worbe, Y., Enander, J., Schreiber, L.R.N., Gillan, C., Fineberg, N.A., Sahakian, B.J., Robbins, T.W., Harrison, N.A., Wood, J., Daw, N.D., Dayan, P., Grant, J.E., Bullmore, E.T., 2015. Disorders of compulsivity: a common bias towards learning habits. Mol Psychiatry 20, 345-352. https://doi.org/10.1038/mp.2014.44

Wang, G.-J., Volkow, N.D., Logan, J., Pappas, N.R., Wong, C.T., Zhu, W., Netusll, N., Fowler, J.S., 2001. Brain dopamine and obesity. The Lancet 357, 354-357. https://doi.org/10.1016/S0140-6736(00)036436

Watson, P., de Wit, S., 2018. Current limits of experimental research into habits and future directions. Current Opinion in Behavioral Sciences 20, 33-39. https://doi.org/10.1016/j.cobeha.2017.09.012

Watson, P., Wiers, R.W., Hommel, B., de Wit, S., 2018. Motivational sensitivity of outcome-response priming: Experimental research and theoretical models. Psychonomic bulletin \& review 25, 2069-2082.

Watson, P., Wiers, R.W., Hommel, B., de Wit, S., 2014. Working for food you don't desire. Cues interfere with goal-directed food-seeking. Appetite 79, 139-148. https://doi.org/10.1016/j.appet.2014.04.005

Watson, P., Wiers, R.W., Hommel, B., Gerdes, V.E.A., de Wit, S., 2017. Stimulus Control Over Action for Food in Obese versus Healthy-Weight Individuals. Frontiers in Psychology 8, 13.

Wong, D.F., Kuwabara, H., Schretlen, D.J., Bonson, K.R., Zhou, Y., Nandi, A., Brašić, J.R., Kimes, A.S., Maris, M.A., Kumar, A., 2006. Increased occupancy of dopamine receptors in human striatum during cue-elicited cocaine craving. Neuropsychopharmacology 31, 2716-2727.

Wood, W., Rünger, D., 2016. Psychology of Habit. Annu. Rev. Psychol. 67, 289-314. https://doi.org/10.1146/annurev-psych-122414-033417

Zhang, Z., Manson, K.F., Schiller, D., Levy, I., 2014. Impaired Associative Learning with Food Rewards in Obese Women. Current Biology 24, 1731-1736. https://doi.org/10.1016/j.cub.2014.05.075 